\title{
Correlações entre Maternidade na Adolescência e Bebê Prematuro e de Baixo Peso: uma revisão integrativa
}

\author{
Gabriela Ribeiro Barros de Faria* \\ Celina Maria Colino Magalhães**
}

\begin{abstract}
Resumo
O presente trabalho apresenta uma revisão integrativa da produção científica nacional e internacional, dos últimos cinco anos, sobre estudos existentes na literatura que abordem a correlação entre a maternidade na adolescência ao nascimento de um bebê prematuro e de baixo peso. Foram acessadas publicações indexadas nas bases de dados Lilacs, Pubmed Central, SciELO (Web of Science), e Scopus (Elsevier), através dos descritores: adolescent, mother, baby ou infant newborn, preterm e low birth weight em artigos de língua inglesa e portuguesa. Entre 206 publicações encontradas, 11 trabalhos foram selecionados de acordo com os critérios de inclusão e por avaliação entre juízes, os quais permitiram compreender temáticas especificas da maternidade adolescente frente ao parto prematuro e de baixo peso ao nascimento. Algumas temáticas foram destacadas como: aspectos emocionais das mães, questões psicossociais, associação entre IMC (índice de massa corporal - peso) materno (pré e peri gestacional) as taxas relativas do nascimento prematuro e ou baixo peso do bebê e o risco da maternidade adolescente e suas comorbidades. Conclui-se que programas de acompanhamento para prevenção da gravidez adolescente, educação em saúde são essenciais para minimizar as taxas de nascimentos prematuros e com baixo peso, assim como a assistência pré-natal direcionada a problemática relativa a condição de saúde do adolescente.

Descritores: Maternidade (Motherhood), Adolescente, Prematuridade, Baixo Peso ao Nascer
\end{abstract}

\section{Correlations between Maternity in Adolescence and the Birth of a Premature and Low Weight Baby: an integrative literature review}

\begin{abstract}
The present work presents an integrative review of the national and international scientific production, of the last five years, on existing studies in the literature that address the correlation between motherhood in adolescence and the birth of a premature and low weight baby. Publications indexed in the Lilacs, Pubmed Central, SciELO (Web of Science), and Scopus (Elsevier) databases were accessed through the descriptors: adolescent, mother, baby or infant newborn, preterm and low birth weight, in English and Portuguese languages. Among 206 publications found, 11 papers were selected according to the inclusion criteria and by evaluation among judges, which allowed to understand specific themes of adolescent motherhood in the face of premature birth and low birth weight. Some themes were highlighted as: emotional aspects of mothers, psychosocial issues, association between maternal BMI (body mass index - weight) (pre and peri gestational), relative rates of premature birth and or low weight of the baby and the risk of motherhood adolescent and his comorbidities. It is concluded that follow-up programs for the prevention of adolescent pregnancy, health education are essential to minimize the rates of premature births and low birth weight, as well as prenatal care directed to problems related to the adolescent's health condition.

Index Terms: Motherhood, Adolescent, Prematurity, Low Birth Weight
\end{abstract}

* Terapeuta Ocupacional. Mestre e doutoranda no Programa de Pós-Graduação em Teoria e Pesquisa do Comportamento da Universidade Federal do Pará. ** Psicóloga, Doutora e Professora do Programa de Pós-graduação em Teoria e Pesquisa do Comportamento da Universidade Federal do Pará. 


\section{Introdução}

A adolescência é uma fase da vida em que se inicia o processo de amadurecimento das características corporais secundárias que, a partir de um fator precipitante, desencadeiam intenso bombardeio hormonal, para atingir a maturidade corporal, especialmente no aspecto reprodutivo. As mudanças durante a adolescência são universais em relação as características físico/corporais. Entretanto, as modificações em nível psicológico e relacional/social dependerão de padrões culturais e ambientais em que o indivíduo vive. A adolescência inspira um momento de decisões individuais e demanda modificações em seu contexto de vida, pelo qual passa de mero observador/espectador para um papel social ativo, questionador, processo essencial para o desenvolvimento de sua personalidade e aperfeiçoamento social (Becker, 1993).

O período cronológico da adolescência ocorre entre os 10 e 19 anos de idade. Por configurar uma fase importante do desenvolvimento humano, visa preparar o organismo à vida adulta, especialmente no aspecto psicossocial, passando da condição de semi-independente para uma autonomia total ou independente (Tiba, 2010). É comum que neste período de transição ocorra o início da vida sexual, que pode refletir em uma gravidez precoce e, possivelmente, indesejada. Conceber durante a adolescência representa indicadores preocupantes de vulnerabilidade tanto à mãe quanto ao bebê. Esse processo oferece riscos à saúde de ambos, bem como prejuízos psicossociais, que podem influenciar negativamente na vida do binômio mãe-bebê. Além, das repercussões familiares, educacionais, sociais, dentre outras condições, como a falta de planejamento e proteção durante a relação sexual, a existência de múltiplos parceiros e o uso de substâncias como o álcool. A gravidez nesta fase implica em ruptura dos projetos sociais, escola e o afastamento de ambientes de interações sociais, condição que pode gerar conflitos diante das mudanças simultâneas próprias da adolescência e da gravidez (Ochen, Chi, \& Lawoko, 2019).

A gravidez na adolescência reflete um sério problema social e de saúde pública em todo o mundo, é multifatorial e está relacionada ao comportamento individual, tradicional, sociocultural ou de natureza religiosa. Além dos correlatos ao baixo status socioeconômico, a educação limitada e a atividade sexual precoce e sem proteção, que implicam em aumentar a condição de vulnerabilidade e de necessidade de uma assistência em saúde diferenciada (Shahabuddin, Nostlinger, Delvaux, Sarker, Delamou, Bardaj1, Broerse, \& Brouwere, 2017).
Entre as gestações e partos registrados anualmente em todo o mundo, $11 \%$ são provenientes de meninas entre 15 - 19 anos, com variações deste percentual de acordo com a região ou cultura (Shahabuddin et al, 2017; Asnong, Fellmeth, Plugge, Wai, Pimanpanarak, Paw, Charunwatthana, Nosten, \& McGready, 2018). Na China este percentual varia entre 2 a 2,5\%, enquanto que na América Latina este percentual pode chegar a 18\% (Sychareun, Vongxay, Houaboun, Thammavongsa, Phummavongsa, Chaleunvong, \& Durham, 2018).

Estima-se que a taxa de morbimortalidade na adolescência seja um terço mais alta do que de mulheres jovens, entre 20 e 25 anos. As principais complicações encontradas durante a gravidez e parto em adolescentes são: distúrbios hipertensivos da gravidez, anemia, diabetes gestacional, ou outras complicações durante o parto. A gravidez na adolescência também pode gerar riscos ao bebê, como nascimento prematuro, baixo peso ao nascimento, doenças respiratórias e maior prevalência de mortalidade infantil (Kane, Miedema, Dielemana, \& Broerse, 2019).

A gravidez dura em média 40 semanas de gestação, com uma variação entre 37 e 42 semanas. O período gestacional é contabilizado a partir da data da última menstruação materna. Considerando o período gestacional, o nascimento prematuro (PT) relaciona-se a uma condição definida de bebês que nascem antes da $37^{a}$ semana de gestação, ou seja, aos que nascem com até 36 semanas e 7 dias. O baixo peso (BP) refere-se ao bebê que nasce com peso abaixo de $2500 \mathrm{~g}$, independente de sua idade gestacional (Mota, Sá, \& Frota, 2005). A prevalência global de bebês com baixo peso ao nascer é de 15,5\% (Ncube, Barlow, \& Mayers, 2016).

Os recém-nascidos PT e BP podem apresentar várias complicações e dificuldades de adaptação a vida extrauterina. Devido a imaturidade dos diversos sistemas orgânicos, quanto menor a idade gestacional e o baixo peso ao nascimento maior tendência as complicações e os riscos para a saúde e manutenção da vida do bebê (Mota, Sá, \& Frota, 2005).

A imaturidade do recém-nascido (RN), PT e BP, o torna propenso a déficits de respostas imunológicas e de função pulmonar, aumentando o risco de morbidade respiratória, longa permanência no ambiente hospitalar, maior frequência de (re)internação, icterícia neonatal, infecções, dificuldades na alimentação e amamentação, bem como complicações neurológicas que podem afetar o desenvolvimento da criança (Machado Jr, Passini Jr, \& Rosa, 2014). $\mathrm{Na}$ maioria dos casa, estes bebês necessitam de intervenção 


\section{E BEBÊ PREMATURO E DE BAIXO PESO: UMA REVISÃO INTEGRATIVA}

médica e apoio tecnológico sistemático, como suporte em unidade neonatal (Mota, Sá, \& Frota, 2005).

Um estudo realizado no Brasil por Leal, Esteves-Pereira, Nakamura-Pereira, Torres, Theme-Filha, Domingues, Dias, Moreira, e Gama (2016), sobre nascimentos entre os anos de 2011 e 2012 ( $\mathrm{n}=23.447$ nascimentos), constatou que os partos prematuros representaram 11,5\% $(n=2.771$ nascimentos) do total dos nascimentos neste período, provenientes de trabalho de parto espontâneo, e apresentam as seguintes características: maior frequência entre mulheres adolescentes; com menor escolaridade; nulíparas; com cuidados pré-natais inadequados; gravidez múltipla; infecções, restrição de crescimento intrauterino; descolamento prematuro da placenta ou má formação do recém-nascido.

A condição PT e BP é comumente associada a hospitalização prolongada do RN, sendo comum desencadear situações de estresse na família, especialmente na mãe, que pode levar a uma disfunção familiar subsequente (Lakshmanan, Agni, Lieu, Flugler, Friedlich, McCormick, \& Belfort, 2017). Sofrem maior incidência de sofrimento psicológico, incluindo medo, ambivalência emocional e falta de confiança em exercer o papel materno. E alguns casos o sofrimento psicológico da mãe aumenta o risco futuro de problemas de desenvolvimento e do comportamento da criança (Ncube, Barlow, \& Mayers, 2016).

As inúmeras demandas médicas e de cuidados com o RN podem durar meses ou anos após a alta neonatal e afetam a qualidade de vida dos pais e da família em geral, com impactos sobre o bem-estar, como o isolamento social e encargos financeiros (Lakshmanan et al, 2017).

Considerando a situação de dupla imaturidade entre o binômio mãe-bebê, de vulnerabilidade e de problemáticas de saúde e de desenvolvimento psicossocial, o objetivo deste artigo é realizar uma revisão integrativa da literatura, correlacionando os dados de pesquisas sobre a gravidez na adolescência e a ocorrência do parto prematuro e/ou de baixo peso ao nascimento. Observa-se a necessidade inerente de compreender a realidade desta problemática na ocorrência de dupla imaturidade e de riscos relativos a saúde da mãe (adolescente) e do bebê (pré-termo e de baixo peso).

\section{Método}

\section{Procedimentos Gerais}

Trata-se de uma revisão integrativa da literatura, na perspectiva de correlacionar estudos que abordem a maternidade adolescente e os nascimentos de bebês pré-termo e de baixo peso, delineando os temas relacionados a este fenômeno considerando os aspectos sociais, psicoafetivos e de saúde.
Para realizar esta revisão, foi utilizado o Modelo de Principais Itens para Relatar Revisões Sistemáticas e Meta Análise (PRISMA), estruturando didaticamente as etapas para a escolha das fontes de dados, a seleção dos descritores, a busca de artigos, a análise dos títulos e resumos, leitura crítica dos artigos na íntegra, a adoção de critérios para inclusão e exclusão, a extração dos dados e a avaliação dos artigos.

A pesquisa foi realizada nas bases Lilacs, PubMed Central, SciELO (Web of Science) e Scopus, acessados através do portal de periódicos da CAPES. Os estudos pesquisados são referentes aos últimos cinco anos de publicação. A busca ocorreu no período de outubro de 2019 a fevereiro de 2020, a partir da combinações dos seguintes descritores: Lilacs: "mãe", "adolescente" e "bebê"; PubMed Central: "mother" AND "adolescente" AND "baby" AND "preterm"; SciELO (Web of Science): "adolescent mother" AND "baby" AND "preterm" AND "low birth weight" e Scopus: "Adolescent" OR «Mother» OR «Infant, Newborn» OR «Prematurity. Os termos preferencialmente, deveriam estar presentes no título e/ou palavras-chave. Ao todo foram encontrados 6.746 trabalhos.

Inicialmente foram acessadas pesquisas da Cochrane Library no sentido de avaliar possíveis revisões relacionadas ao tema em estudo, foram encontrados dois trabalhos com períodos acima de cinco anos de publicação.

Os critérios de inclusão nesta revisão foram: 1) tratar-se de artigos revisados por pares, 2) estar escrito nos idiomas inglês ou português até 5 anos de publicação, 3) artigos que fizessem referência a maternidade na adolescência correlata ao nascimento de um bebê prematuro e de baixo peso, 4) artigos na íntegra e de acesso livre.

Como critérios de exclusão: 1) artigos não revisados por pares, 2) artigos de revisão ou resenhas, 3) trabalhos escritos em outras línguas que não o português ou inglês, 4) artigos teóricos relacionados ao tema, 5) artigos não disponíveis na íntegra e não gratuitos e 6) estudos que não se relacionem com o tema específico.

A revisão integrativa seguiu um modelo criterioso de registro de dados, a partir de seis fases distintas: elaboração da pergunta norteadora, busca na literatura, coleta de dados, análise crítica dos estudos incluídos, discussão dos resultados e elaboração da revisão integrativa. Os dados coletados dos trabalhos foram detalhados em um instrumento validado de Ursi (2005) apud Souza, Silva, e Carvalho (2010), que inclui título, periódico, autor (es), idioma, ano de publicação, instituição de estudo, tipo de publicação, objetivos, amostra, tratamento dos dados, resultados, análise e implicações. Nas etapas desta revisão, participaram dois avaliadores independentes, sendo incluídos os estudos que apre- 
sentaram concordância, com índice de confiabilidade acima de $75 \%$.

\section{Procedimentos de Análise}

Os artigos encontrados $(\mathrm{N}=6.746)$ foram avaliados previamente através de leitura dinâmica do título e da descrição, realizando uma categorização para o tema e a população de estudo. Em seguida, foram selecionados 206 artigos para uma análise mais específica sobre a maternidade adolescente e os desfechos desta gestação que se correlacionassem ao nascimento de um bebê pré-termo e de baixo peso.

\section{Resultados}

A síntese dos resultados obtidos nas etapas de triagem dos artigos é apresentada na figura 1; considerando os itens de inclusão e exclusão. Foram analisados os resumos dos 206 artigos, sendo selecionados 11 artigos que atenderam os objetivos da pesquisa, de acordo com os critérios de elegibilidade e relevância entre juízes. Os demais artigos (195 artigos) foram excluídos pelo fato de não se relacionarem o tema deste estudo.

No quadro 1 estão listados os artigos selecionados e seus respectivos dados, descrevendo autores, ano de publicação do estudo, país onde foi realizado, idioma de publicação, objetivos, população de estudo e aspectos metodológicos.

Dos 11 artigos incluídos, 7 foram publicados exclusivamente na língua inglesa, 3 exclusivamente na língua portuguesa, e $1 \mathrm{em}$ com versão nas duas línguas. Entre o local em que foram realizados os estudos, seis trabalhos foram em países em desenvolvimento, incluindo o Brasil, quatro em países desenvolvidos e um estudo misto, com dados de ambos (em desenvolvimento e desenvolvidos).

Os dados dos estudos mostram que todos correlacionaram as situações da gravidez na adolescência ao nascimento prematuro e/ou de baixo peso. Entre os 11 trabalhos encontrados, apenas dois realizaram um estudo empírico e qualitativo, que envolviam a percepção da mãe sobre a condição de ser adolescente e da condição de saúde do seu bebê; os demais abordaram estudos retrospectivos ou de coorte para definir as associações estatísticas a dados pessoais do histórico materno (condições sociodemográficas, familiares, peso, gestação e parto) ao risco do nascimento prematuro e/ou de baixo peso, considerando a situação de vulnerabilidade social e de saúde que envolve o binômio mãe-bebê.

\section{Figura 1. Distribuição das publicações conforme base de dados}

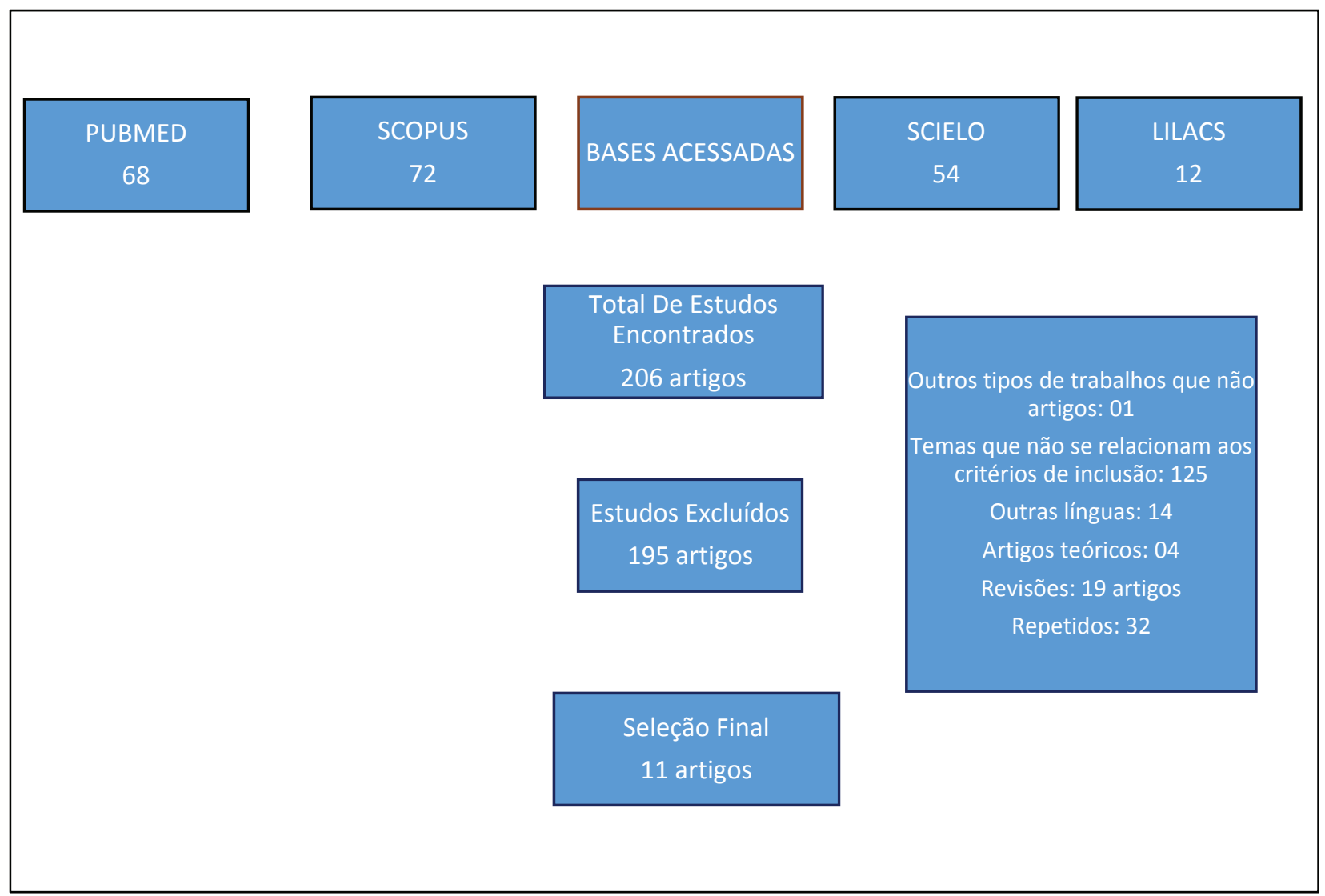

Fonte: Adaptado de PRISMA 2009 FlowDiagram (Moher, Liberati, Tetzlaff \& Altman, 2009) 
E BEBÊ PREMATURO E DE BAIXO PESO: UMA REVISÃO INTEGRATIVA

\section{Quadro 1. Estudos que correlacionam as vivências da maternidade adolescente com a criança pré-termo e de baixo peso, descritos nos últimos cinco anos.}

\begin{tabular}{|c|c|c|c|c|c|}
\hline Autor (ano) & Local & $\begin{array}{l}\text { Idioma } \\
\text { Publicado }\end{array}$ & Objetivos & População de estudo & Delineamento \\
\hline $\begin{array}{l}\text { Barroso, Pontes, e Rolim } \\
(2015)\end{array}$ & Brasil & Português & $\begin{array}{l}\text { Apreender sob percepção materna as } \\
\text { consequências da internação em Unidade } \\
\text { de Terapia Intensiva Neonatal no } \\
\text { estabelecimento do vínculo afetivo mãe } \\
\text { adolescente/bebê prematuro }\end{array}$ & $\begin{array}{l}10 \text { mães adolescentes } \\
\text { de bebês pré-termo e de } \\
\text { baixo peso internados }\end{array}$ & $\begin{array}{l}\text { Estudo exploratório-descritivo, } \\
\text { de natureza qualitativa }\end{array}$ \\
\hline $\begin{array}{l}\text { Sámano, Martínez-Rojano, } \\
\text { Chico-Barba, Sánchez- } \\
\text { Jiménez, Illescas-Zarate, e } \\
\text { Rodríguez-Ventura (2019) }\end{array}$ & México & Inglês & $\begin{array}{l}\text { Analisar a associação entre as características } \\
\text { da rede de suporte familiar (tamanho e } \\
\text { membros) e os resultados da gravidez em } \\
\text { adolescentes. }\end{array}$ & $\begin{array}{l}352 \text { adolescentes } \\
\text { entre } 12 \text { a } 18 \text { anos, } \\
\text { organizadas em quatro } \\
\text { grupos. }\end{array}$ & $\begin{array}{l}\text { Estudo transversal, realizado } \\
\text { entre } 2008 \text { e 2014, com } \\
\text { coleta de dados do pré-natal } \\
\text { no Instituto Nacional de } \\
\text { Perinatologia (Instituto Nacional } \\
\text { de Perinatologia }\end{array}$ \\
\hline $\begin{array}{l}\text { Chvatal, Vasconcellos, } \\
\text { Rivoredo, e Turato (2017) }\end{array}$ & Brasil & Português & $\begin{array}{l}\text { Desvelar os sentimentos e os mecanismos } \\
\text { de defesa vivenciados no puerpério por } \\
\text { adolescentes primíparas cujos bebês } \\
\text { prematuros foram internados em Unidade } \\
\text { Neonatal (UTI) }\end{array}$ & $\begin{array}{l}07 \text { mães adolescentes } \\
\text { com idades entre } 13 \text { e } \\
19 \text { anos. }\end{array}$ & Caracterização clínico-qualitativa \\
\hline $\begin{array}{l}\text { Santos, Costa, Amaral, } \\
\text { Vieira, Bacelar, e Almeida } \\
\text { (2014) }\end{array}$ & Brasil & Português & $\begin{array}{l}\text { Avaliar a associação entre a gravidez de } \\
\text { adolescentes }<16 \text { anos e a ocorrência de } \\
\text { nascidos vivos de baixo peso, prematuridade } \\
\text { e cesariana. }\end{array}$ & $\begin{array}{l}19.869 \text { nascidos vivos } \\
\text { de mães adolescentes } \\
(10-19 \text { anos) utilizando } \\
\text { o sistema de infor- } \\
\text { mações (SISNAC) no } \\
\text { período } 2006 \text { a } 2012 .\end{array}$ & $\begin{array}{l}\text { Estudo transversal, que } \\
\text { utilizou dados do sistema de } \\
\text { informações de nascidos vivos } \\
\text { (SISNAC/DATASUS), no } \\
\text { período de } 2006 \text { a } 2012\end{array}$ \\
\hline $\begin{array}{l}\text { Belfort, Santos, Pessoa, } \\
\text { Dias, Heidelmann, e } \\
\text { Saunders (2018) }\end{array}$ & Brasil & $\begin{array}{l}\text { Português e } \\
\text { inglês }\end{array}$ & $\begin{array}{l}\text { Identificar, através de modelagem } \\
\text { hierarquizada, os fatores que determinam } \\
\text { a ocorrência do baixo peso ao nascer } \\
\text { em filhos de adolescentes, atendidas em } \\
\text { maternidade pública. }\end{array}$ & $\begin{array}{l}751 \text { adolescentes e seus } \\
\text { respectivos filhos. }\end{array}$ & Modelagem hierarquizada \\
\hline $\begin{array}{l}\text { Hoffman, Bann, Higgins, e } \\
\text { Vohr (2015) }\end{array}$ & $\begin{array}{l}\text { Estados } \\
\text { Unidos }\end{array}$ & Inglês & $\begin{array}{l}\text { Avaliar os resultados cognitivos, de } \\
\text { linguagem e de comportamento de bebês } \\
\text { EPT nascidos de mães adolescentes em } \\
\text { comparação com bebês de mães mais velhas. } \\
\text { Bem como explorar as construções sociais e } \\
\text { domésticas. }\end{array}$ & $\begin{array}{l}211 \text { Bebês de mães } \\
\text { adolescentes extre- } \\
\text { mamente prematuros } \\
\text { (EPT) (>27 semanas) } \\
\text { de } 18 \text { a } 22 \text { meses. }\end{array}$ & $\begin{array}{l}\text { Estudo de coorte entre os anos } \\
\text { de } 2002 \text { a } 2011\end{array}$ \\
\hline $\begin{array}{l}\text { Houde, Dahdouh, } \\
\text { Mongrain, Dubuc, } \\
\text { Francoeur, e Balayla (2015) }\end{array}$ & $\begin{array}{l}\text { Estados } \\
\text { Unidos }\end{array}$ & Inglês & $\begin{array}{l}\text { Descrever as tendências de distribuição do } \\
\text { IMC (diretrizes do Instituto de Medicina } \\
\text { - OIM) na pré-gestação e ganho de peso } \\
\text { durante a gravidez em adolescentes e em } \\
\text { adultas; e determinar se padrões de risco } \\
\text { obstétrico e neonatal ( PTB e BPN) são } \\
\text { observados. }\end{array}$ & $\begin{array}{l}\text { 1,036,346 entre } \\
\text { adolescentes e adultas. }\end{array}$ & $\begin{array}{l}\text { Estudo de coorte retrospec- } \\
\text { tivo, de base populacional, } \\
\text { usando dados estatísticos } \\
\text { vitais dos "Arquivos de Dados } \\
\text { de Nascimento" do Centro } \\
\text { Nacional de Estatísticas de } \\
\text { Saúde (Centros de Controle } \\
\text { e Prevenção de Doenças). } \\
\text { Dados para } 2012 \text { com os } \\
\text { registros dos Estados Unidos, } \\
\text { Havaí e Alasca. }\end{array}$ \\
\hline $\begin{array}{l}\text { Restrepo-Méndez, Lawlor, } \\
\text { Horta, Matijasevich, Santos, } \\
\text { Menezes, Barros, e Victora } \\
(2015)\end{array}$ & $\begin{array}{l}\text { Inglaterra } \\
\text { e Brasil }\end{array}$ & Inglês & $\begin{array}{l}\text { Explorar as associações da idade materna } \\
\text { com o BPN e o parto prematuro em } \\
\text { relação posição socioeconômica com a } \\
\text { morbimortalidade infantil e com resultados } \\
\text { adversos ao longo da vida. }\end{array}$ & $\begin{array}{l}7680 \text { nascimentos de } \\
\text { mães adolescentes nos } \\
\text { anos de 1982, } 1993 \text { e } \\
2004 \text { em Pelotas, Brasil, } \\
\text { e no Reino Unido } \\
\text { em } 1991 \text { - Estudo } \\
\text { Longitudinal de Pais } \\
\text { e Filhos da Avon } \\
\text { (ALSPAC)]. }\end{array}$ & $\begin{array}{l}\text { Coortes de tempo, nos anos de } \\
1982,1993 \text { e } 2004 \text { do Brasil; e } \\
\text { do ano de } 1990 \text { de uma cidade } \\
\text { do sudoeste da Inglaterra } \\
\text { (Estudo longitudinal da Avon de } \\
\text { pais e Crianças - ALSPAC) }\end{array}$ \\
\hline Baker, e Haeri (2014) & $\begin{array}{l}\text { Estados } \\
\text { Unidos }\end{array}$ & Inglês & $\begin{array}{l}\text { Identificar fatores de risco potencialmente } \\
\text { modificáveis para o nascimento prematuro } \\
\text { espontâneo em uma população adolescente } \\
\text { americana contemporânea. }\end{array}$ & $\begin{array}{l}650 \text { mães adolescentes } \\
\text { nulíparas, com idades } \\
\text { entre } 11 \text { e } 18 \text { anos }\end{array}$ & $\begin{array}{l}\text { Análise de coorte de } 2000 \text { a } \\
\text { 2004, em um Centro Hospitalar } \\
\text { de Washington }\end{array}$ \\
\hline $\begin{array}{l}\text { Kirbas, Gulerman, e Daglar } \\
\text { (2016) }\end{array}$ & Turquia & Inglês & $\begin{array}{l}\text { Investigar se há um risco de complicações } \\
\text { perinatais nas gestações de adolescentes e } \\
\text { a relação entre gravidez na adolescência e } \\
\text { parto prematuro. }\end{array}$ & $\begin{array}{l}582 \text { pacientes } \\
\text { adolescentes grávidas } \\
\text { (entre } 15 \text { e } 19 \text { anos) e } \\
2.920 \text { mulheres (entre } \\
20 \text { e } 34 \text { anos) }\end{array}$ & $\begin{array}{l}\text { Coorte retrospectiva do tipo } \\
\text { caso-controle so }\end{array}$ \\
\hline $\begin{array}{l}\text { Botura, Bersani-Amado, } \\
\text { Teixeira, Vituri, Caparroz- } \\
\text { Assef, e Cuman (2018) }\end{array}$ & Brasil & Inglês & $\begin{array}{l}\text { Avaliar a influência da gravidez na } \\
\text { adolescência na ocorrência de baixo peso } \\
\text { ao nascer, prematuridade e óbito em recém- } \\
\text { nascidos internados em UTIN }\end{array}$ & $\begin{array}{l}706 \mathrm{RN}, 192 \text { de mães } \\
\text { adolescentes e } 514 \text { de } \\
\text { mães não-adolescentes } \\
\text { ou adultas }\end{array}$ & $\begin{array}{l}\text { Caracterização descritiva, } \\
\text { exploratória, retrospectivo e de } \\
\text { caráter comparativo }\end{array}$ \\
\hline
\end{tabular}




\section{Quadro 2: descrição dos estudos segundo os instrumentos, as variáveis, os resultados e as temáticas abordadas}

\begin{tabular}{|c|c|c|c|c|}
\hline Autor (ano) & $\begin{array}{l}\text { Instrumentos } \\
\text { utilizados }\end{array}$ & Dados de análise & Principais Resultados & Temáticas \\
\hline $\begin{array}{l}\text { Barroso, Pontes, } \\
\text { e Rolim (2015) }\end{array}$ & $\begin{array}{l}\text { Entrevista semi- } \\
\text { estruturada e diário de } \\
\text { campo }\end{array}$ & $\begin{array}{l}\text { Dados pessoais: faixa etária, estado civil, } \\
\text { escolaridade, procedência e número de filhos. } \\
\text { Questões de pesquisa: sentimentos } \\
\text { vivenciados na descoberta da prematuridade } \\
\text { e internação dos bebês. } \\
\text { Observação livre para perceber formas de } \\
\text { estabelecimento do vínculo mãe bebê, como: } \\
\text { troca de olhar, toque carinhoso, palavras de } \\
\text { conforto e gesto de acalanto }\end{array}$ & $\begin{array}{l}\text { Pôde-se perceber pelas falas que a experiência } \\
\text { dolorosa de gerar um filho com algum problema } \\
\text { grave de saúde, prematuro ou de baixo peso, } \\
\text { desencadeia, na maioria das vezes, um processo de } \\
\text { estresse e crises psicológicas na mãe e na família. } \\
\text { Surge, então, sentimento de culpa, e/ou ambivalência } \\
\text { para com a criança. Com isso a maternidade parece } \\
\text { se modificar, dependendo da necessidade que surge } \\
\text { com o problema do bebê de risco }\end{array}$ & $\mathrm{A}$ e $\mathrm{B}$ \\
\hline $\begin{array}{l}\text { Sámano et al } \\
(2019)\end{array}$ & $\begin{array}{l}\text { Formulário de dados } \\
\text { pessoais da adolescente } \\
\text { grávida e rede social de } \\
\text { apoio }\end{array}$ & $\begin{array}{l}\text { Formas de apoio social, nome, idade, estado } \\
\text { civil, nível de educação, ocupação, número } \\
\text { de pessoas na casa, tipo de família, número } \\
\text { de crianças e adolescentes que moram com } \\
\text { a família. } \\
\text { Peso pré e peri-gestacional, altura, IMC. } \\
\text { Idade gestacional e peso do bebê. } \\
\text { Nível socioeconômico (condições de renda } \\
\text { da família) }\end{array}$ & $\begin{array}{l}\text { A idade média foi de } 15 \pm 1 \text { ano e a idade gine- } \\
\text { cológica foi de } 4 \text { anos. A maioria dos participan- } \\
\text { tes vieram de famílias nucleares, eram de nível } \\
\text { socioeconomico muito baixo, baixo e médio; e } \\
\text { tinham menos de } 9 \text { anos de estudo. As adoles- } \\
\text { centes do grupo } 3 \text { tiveram maior frequência de } \\
\text { se casar ou coabitar, o peso e o comprimento } \\
\text { dos recém-nascidos foram menores, era comum } \\
\text { encontrar crianças menores de } 15 \text { anos na família } \\
\text { e houve maior participação da sogra. }\end{array}$ & $B, C$ e $D$ \\
\hline $\begin{array}{l}\text { Chvatal, } \\
\text { Vasconcellos, } \\
\text { Rivoredo, e } \\
\text { Turato (2017) }\end{array}$ & $\begin{array}{l}\text { Entrevista semidirigida } \\
\text { e registro em diário de } \\
\text { campo. }\end{array}$ & $\begin{array}{l}\text { Características sócio-demográficas: idade, } \\
\text { situação conjugal, ocupação, escolarização, } \\
\text { com quem mora. } \\
\text { Perguntas abertas e dados biodemográficos } \\
\text { obtidos do próprio relato das participantes. } \\
\text { Observações do comportamento global e } \\
\text { linguagem não-verbal }\end{array}$ & $\begin{array}{l}\text { Das sete adolescentes quatro eram solteiras, duas } \\
\text { casadas e uma separada. Cinco cursando o Ensi- } \\
\text { no Fundamental (EF) e duas com EF incompleto. } \\
\text { As descrições do plano psicológico expressaram } \\
\text { a dor frente à impossibilidade de estar com o } \\
\text { filho e o sentimento de que não é possível, num } \\
\text { contexto como o da internação. }\end{array}$ & $A$ e $B$ \\
\hline $\begin{array}{l}\text { Santos et al } \\
(2014)\end{array}$ & $\begin{array}{l}\text { Consulta aos dados } \\
\text { sociodemográficos e aos } \\
\text { relacionados à mãe e } \\
\text { ao bebê }\end{array}$ & $\begin{array}{l}\text { Variáveis sociodemográficas: faixa etária } \\
\text { materna, estado civil, escolaridade; } \\
\text { relacionadas à gestação e parto (número de } \\
\text { consultas pré-natal, tipo de parto - normal } \\
\text { ou cesariano); relacionadas ao RN (peso de } \\
\text { nascimento e idade gestacional). }\end{array}$ & $\begin{array}{l}19,5 \% \text { do total de nascimentos foram de mães } \\
\text { adolescentes; a maioria era solteira; com segundo } \\
\text { grau e a faixa de mães }<16 \text { anos representou } \\
30,2 \% \text {. Maioria realizou o pré-natal com número } \\
\text { insuficiente de consultas, e } 13,5 \% \text { dos } \mathrm{RN} \text { foram } \\
\text { prematuros e } 12,2 \% \text { de baixo peso. Os achados } \\
\text { mostraram associação estatisticamente significante } \\
\text { entre faixa etária materna }<16 \text { anos e RN com } \\
\text { baixo peso e nascimento prematuro. }\end{array}$ & $B$ e $D$ \\
\hline $\begin{array}{l}\text { Belfort et al } \\
\text { (2018) }\end{array}$ & $\begin{array}{l}\text { Consulta aos } \\
\text { prontuários das } \\
\text { adolescentes e recém } \\
\text { nascidos e entrevista } \\
\text { realizada durante as } \\
\text { consultas de nutrição no } \\
\text { pré-natal. }\end{array}$ & $\begin{array}{l}\text { Fatores sócio-demográficos: local de moradia, } \\
\text { condições de saneamento, idade materna, } \\
\text { situação marital, cor da pele, escolaridade, } \\
\text { renda familiar, número de pessoas na família, } \\
\text { planejamento e aceitação da gestação. } \\
\text { Pré-natal: número de consultas, idade } \\
\text { gestacional da primeira consulta pré-natal, } \\
\text { grupo de estudo. } \\
\text { Fatores da adolescente: menarca, idade } \\
\text { ginecológica, número de gestações, intervalo } \\
\text { intergestacional, estado nutricional pré- } \\
\text { gestação, ganho de peso gestacional, anemia, } \\
\text { cegueira noturna, intercorrências, síndromes } \\
\text { hipertensivas, duração da gestação e uso de } \\
\text { drogas. } \\
\text { Baixo peso do bebê. }\end{array}$ & $\begin{array}{l}\text { Dez por cento dos recém-nascidos apresenta- } \\
\text { ram BPN e } 13 \% \text { tiveram nascimento prematuro. } \\
\text { A média de idade no parto foi de } 17,5 \text { anos } \pm \\
1,6 \text {, maioria ensino fundamental completo, não } \\
\text { trabalhavam, eram solteiras, se autodeclararam } \\
\text { como não brancas, apresentaram renda per capita } \\
\text { inferior a um salário mínimo e tinham acesso } \\
\text { a saneamento básico adequado. As variáveis } \\
\text { idade materna, ausência de situação marital, não } \\
\text { aceitação da gravidez e início tardio do pré-natal } \\
\text { associaram-se ao BPN. }\end{array}$ & $\mathrm{B}, \mathrm{C}$ e $\mathrm{D}$ \\
\hline $\begin{array}{l}\text { Hoffman, Bann, } \\
\text { Higgins, e Vohr } \\
(2015)\end{array}$ & $\begin{array}{l}\text { Exame neurológico } \\
\text { completo, Bayley-III } \\
\text { e BITSEA (Avaliação } \\
\text { Emocional Social da } \\
\text { Criança Pequena) }\end{array}$ & $\begin{array}{l}\text { Bayley-III para pontuações cognitivas de } \\
\text { linguagem e motoras. } \\
\text { Avaliação Emocional Social da Criança } \\
\text { Pequena (BITSEA / P), com escala de } \\
\text { problemas e uma escala de competência. } \\
\text { Características sociais, pessoais e clínicas da } \\
\text { mãe e da criança. }\end{array}$ & $\begin{array}{l}\text { As mães adolescentes eram mais frequentemente } \\
\text { solteiras, hispânicas, menos instruídas e possuíam } \\
\text { seguro público. } \\
\text { Os bebês com baixo peso ao nascer de mães } \\
\text { adolescentes apresentam altos riscos sociais e } \\
\text { ambientais associados a resultados adversos de } \\
\text { comportamento. }\end{array}$ & $\mathrm{B}$ e D \\
\hline $\begin{array}{l}\text { Houde et al } \\
(2015)\end{array}$ & $\begin{array}{l}\text { Dados estatísticos dos } \\
\text { "Arquivos de Dados } \\
\text { de Nascimento" do } \\
\text { Centro Nacional de } \\
\text { Estatísticas de Saúde } \\
\text { (Centros de Contro- } \\
\text { le e Prevenção de } \\
\text { Doenças), de } 2012 \\
\text { com os registros dos } \\
\text { Estados Unidos, Havaí } \\
\text { e Alasca. } \\
\text { Avaliação do Índice } \\
\text { de Massa Corporal } \\
\text { (IMC) de acordo com a } \\
\text { classificação da OMS. }\end{array}$ & $\begin{array}{l}\text { As categorias de IMC (OMS): baixo peso } \\
\text { (menor que } 18,5) \text {, normal }(18,5 \text { a } 24,9) \text {, } \\
\text { excesso de peso }(25,0 \text { a } 25,9) \text {, classe de } \\
\text { obesidade I }(30,0 \text { a } 34,9) \text {, classe de obesi- } \\
\text { dade II }(35,0 \text { a } 39,9) \text { e classe de obesidade } \\
\text { III (maior que 40,0). } \\
\text { Dados sócio-demográficos e clínicos: raça, } \\
\text { paridade, adequação do pré-natal, estado } \\
\text { civil, educação materna, tabagismo, álcool, } \\
\text { antecedentes obstétricos e de parto ante- } \\
\text { riores (número de Cesareas e baixo peso } \\
\text { ao nascimento), idade gestacional ao parto } \\
\text { e peso ao nascer e comorbidades médicas } \\
\text { maternas (distúrbios renais, cardíacos, } \\
\text { diabéticos e hipertensos). }\end{array}$ & $\begin{array}{l}\text { Em relação aos adultos, os adolescentes eram mais } \\
\text { propensos a serem afro-americanos, nulíparos, com } \\
\text { menor nível educacional e não casados. } \\
\text { De acordo com o IMC e o ganho de peso } \\
\text { gestacional, as chances de BPN foram } \\
\text { significativamente maiores para as categorias mais } \\
\text { baixas de IMC e diminuíram para diferenças não } \\
\text { significativas nos grupos obesos }\end{array}$ & $\mathrm{B}, \mathrm{C}$ e $\mathrm{D}$ \\
\hline
\end{tabular}




\begin{tabular}{|c|c|c|c|c|}
\hline Autor (ano) & $\begin{array}{l}\text { Instrumentos } \\
\text { utilizados }\end{array}$ & Dados de análise & Principais Resultados & Temáticas \\
\hline $\begin{array}{l}\text { Restrepo-Méndez } \\
\text { et al (2015) }\end{array}$ & $\begin{array}{l}\text { Quatro coortes: três } \\
\text { coortes de diferentes } \\
\text { períodos de Pelotas, } \\
\text { Brasil (país de renda } \\
\text { média) e uma coorte de } \\
\text { gravidez do sudoeste da } \\
\text { Inglaterra (país de alta } \\
\text { renda) (ALSPAC). }\end{array}$ & $\begin{array}{l}\text { Idade materna, dados da idade gestacional e } \\
\text { peso do bebê ao nascimento. } \\
\text { Dados da posição sócio econômica: renda } \\
\text { familiar, educação materna e educação } \\
\text { paterna - e cor da pele materna / origem } \\
\text { étnica, estado civil e paridade. }\end{array}$ & $\begin{array}{l}\text { A prevalência de BPN tendeu a ser mais alta } \\
\text { entre adolescentes nas coortes de Pelotas e entre } \\
\text { mães com mais de } 34 \text { anos na ALSPAC. A pre- } \\
\text { valência de BPN diminuiu em Pelotas em quase } \\
\text { todas as faixas etárias maternas. A prevalência } \\
\text { de prematuridade foi maior entre adolescentes } \\
\text { e mães com mais de } 34 \text { anos. Em Pelotas, a } \\
\text { prevalência de nascimentos prematuros tendeu a } \\
\text { aumentar ao longo do tempo. }\end{array}$ & $B$ e $D$ \\
\hline $\begin{array}{l}\text { Baker, e Haeri } \\
(2014)\end{array}$ & $\begin{array}{l}\text { Dados demográficos } \\
\text { extraídos do pré-natal } \\
\text { e acompanhamento do } \\
\text { ganho de peso durante a } \\
\text { gravidez }\end{array}$ & $\begin{array}{l}\text { Idade materna, raça / etnia, altura e peso pré- } \\
\text { gestacional, tabagismo, uso de substâncias } \\
\text { ilícitas e data do último período menstrual. } \\
\text { Idade gestacional, IMC peri-gestacional, } \\
\text { registro de doença sexualmente transmissível. }\end{array}$ & $\begin{array}{l}\text { Dos } 650 \text { partos incluídos, } 88 \text { mulheres tiveram } \\
\text { um parto prematuro espontâneo. Havia } 578 \text { mães } \\
\text { negras, } 67 \text { mães hispânicas. } 82 \% \text { tinham IMC } \\
>25 \mathrm{~kg} / \mathrm{m} 2 \text { (excesso de peso). } \\
\text { Mães adolescentes com baixo IMC tiveram com } \\
\text { parto prematuro espontâneo. A obesidade materna } \\
\text { e o ganho de peso acima dos níveis recomendados } \\
\text { pelo Instituto de Medicina (OIM) protegiam contra } \\
\text { o nascimento prematuro espontâneo. }\end{array}$ & B e $C$ \\
\hline $\begin{array}{l}\text { Kirbas, } \\
\text { Gulerman, e } \\
\text { Daglar } \\
(2016)\end{array}$ & $\begin{array}{l}\text { Informações clínicas } \\
\text { obtidas nos prontuários } \\
\text { médicos e/ou } \\
\text { entrevistas por telefone. }\end{array}$ & $\begin{array}{l}\text { Idade, status do seguro público e ganho de } \\
\text { peso materno durante a gravidez e IMC } \\
\text { pré-gestacional. Parâmetros de resultados } \\
\text { perinatais, incluindo idade gestacional no } \\
\text { parto, peso ao nascer e percentil de peso } \\
\text { ao nascer, parto prematuro espontâneo, } \\
\text { mortalidade perinatal, índice de Apgar } 5 \\
\text { minutos } 7 \text { e admissão em unidade de terapia } \\
\text { intensiva neonatal. }\end{array}$ & $\begin{array}{l}\text { As idades médias dos grupos de adolescentes e } \\
\text { controle foram de } 17,9 \text { e } 25,4 \text { anos, respectiva- } \\
\text { mente. A idade gestacional média dos grupos de } \\
\text { adolescentes e controle na primeira consulta pré- } \\
\text {-natal foi de } 11,2 \text { e } 8,5 \text { semanas, respectivamente. } \\
\text { Os riscos de parto prematuro e pré-eclâmpsia } \\
\text { foram significativamente maiores entre as mães } \\
\text { adolescentes. Os bebês destas apresentaram } \\
\text { maiores riscos de baixo peso ao nascer, prema- } \\
\text { turidade, baixo índice de Apgar e internação em } \\
\text { unidade de terapia intensiva neonatal do que os } \\
\text { grupo controle. }\end{array}$ & $B$ e $D$ \\
\hline $\begin{array}{l}\text { Botura et al } \\
(2018)\end{array}$ & $\begin{array}{l}\text { Dados de nascimentos } \\
\text { em um hospital } \\
\text { universitário de } \\
\text { referência em média e } \\
\text { alta complexidade. }\end{array}$ & $\begin{array}{l}\text { Idade da mãe - mães adolescentes }(10 \text { a } \\
19 \text { anos) e mães sem filhos ou adultos }(> \\
20 \text { anos); idade gestacional (semanas) - } \\
\text { prematuro (<37 semanas) e RN a termo } \\
\text { ( }>37 \text { semanas); peso ao nascer (gramas) } \\
\text { - considerando baixo peso o RN }<2500 \\
\text { g, e não abaixo do peso o RN } \geq 2500 \text { g; e } \\
\text { resultado - pela ocorrência ou não de morte } \\
\text { do RN. Para avaliar possíveis associações - } \\
\text { Risco Relativo: entre a idade da mãe e o peso } \\
\text { ao nascer, prematuridade e desfecho (óbito). }\end{array}$ & $\begin{array}{l}\text { A idade média das mães adolescentes foi de } 16,9 \\
\text { anos. Mães não adolescentes ou adultas, a idade } \\
\text { média foi de } 27,4 \text { anos. Entre os recém-nascidos } \\
\text { admitidos, } 79 \% \text { eram prematuros }(558) \text { e } 72,6 \% \\
\text { apresentavam baixo peso ao nascer }(513) \text {. RNs } \\
\text { prematuros e como o baixo peso foi significativa- } \\
\text { mente maior para as mães adolescentes. } \\
\text { Associações entre idade da mãe, baixo peso ao } \\
\text { nascer, prematuridade, o risco relativo evidenciou } \\
\text { maior probabilidade de morte para o RN de mães } \\
\text { adolescentes internadas na UTIN }\end{array}$ & $\mathrm{D}$ \\
\hline
\end{tabular}

Temáticas: $\mathrm{A}$ - aspectos emocionais das mães, bem como o envolvimento afetivo desta com o bebê; $\mathrm{B}$ - questões psicossociais da gravidez na adolescência; $\mathrm{C}$ - a associação entre IMC materno (índice de massa corporal - peso) às taxas relativas do nascimento prematuro e/ou baixo peso do bebê e $\mathrm{D}$ - risco da maternidade adolescente para a prevalência do nascimento de um bebê pré-termo e/ou de baixo peso e suas comorbidades.

O quadro 2 apresenta os resultados dos estudos identificados sobre a maternidade adolescente e seu bebê PT e BP, os instrumentos utilizados para a coleta de dados e as variáveis analisadas, bem como os resultados de cada estudo.

A respeito dos instrumentos utilizados, dois estudos que fizeram abordagem direta às mães adolescentes utilizaram entrevista semiestruturada ou semidirigida; os demais coletaram os dados a partir do registro em prontuário ou protocolo de avaliação para definir as correlações existentes entre as variáveis maternas e do bebê.

Entre os trabalhos selecionados para esta revisão, alguns se referiram aos aspectos emocionais das mães, bem como o envolvimento afetivo desta com o bebê; as questões psicossociais da gravidez na adolescência; outros relacionaram ao estudo do índice de massa corporal (IMC) da mãe (antes e durante a gestação) com a preva- lência relativa ao nascimento de um bebê PT e com BP. Outro estudo apontou as possíveis comorbidades em saúde do bebê.

\section{Discussão}

O nascimento prematuro e com baixo peso tem sido amplamente descrito na literatura como uma condição influente para o aumento da morbimortalidade infantil, em virtude das consequências que isto acarreta nas condições de saúde da criança (Oliveira, Santos, \& Melo, 2016; Silveira, Matijazevit, Horta, \& Bettiol, 2013; Von Grafen, Gomes, Lorenzoni, Strassburger, Ferreira, \& Campanaro, 2017). Uma das possíveis causas do aumento desta prevalência de partos prematuros e com baixo peso reporta-se a idade materna e aos fatores psicossociais relativos à maternidade (Flores-Valencia, Nava-Chapa, \& Arenas-Monreal, 
2017; Kawakita, Wilson, Grantz, Landy, Huang, \& Gomes-Lobo, 2016).

A adolescência se configura em um período de intensas mudanças físico-corporais da mãe, assim como nos aspectos psicológicos e afetivos, influenciados pelo estilo de vida e pelos contextos culturais e familiares do indivíduo. A maternidade adolescente e o nascimento prematuro e com baixo peso de um bebê podem potencialmente influenciar nos resultados de saúde e nas condições de vulnerabilidade e risco social no binômio mãe-bebê, uma vez que se formaliza a existência de uma dupla imaturidade (Azevedo, Diniz, Fonseca, Azevedo, \& Evangelista, 2015).

Este trabalho teve como objetivo apresentar pesquisas, dos últimos cinco anos, que identifiquem a correlação entre a maternidade adolescente e o bebê com nascimento prematuro e de baixo peso.

A procura de artigos pelos descritores oportunizou compreender a dimensão das pesquisas que são realizadas considerando a maternidade, a adolescência e o recém-nascido. Por um lado, estudos qualitativos permitiram a compreensão de alguns aspectos emocionais envolvidos sobre a contextualização da maternidade adolescente frente a uma bebê frágil e que necessita de cuidados específicos em saúde; por outro lado, os estudos enfatizaram a importância de estudar algumas variáveis relacionadas a prevalência do baixo peso e da prematuridade provenientes de uma gravidez precoce.

O meio de acesso aos dados, predominante na maioria dos estudos selecionados nesta revisão, tiveram origem em um levantamento retrospectivo, seja no histórico do pré-natal materno, no prontuário da unidade materno-infantil ou em protocolos específicos de acompanhamento do bebê.

Independente do meio em que os dados foram coletados, qualitativos e quantitativos, a relevância das pesquisas descritas nesta revisão reporta a temáticas específicas, que melhor descrevem as correlações entre o contexto de uma maternidade na adolescência na condição do nascimento de um bebê PT e de BP.

\section{Temática $A$ - aspectos emocionais das mães e o envolvimento afetivo desta com o bebê}

Referem-se aos estudos que delinearam os sentimentos maternos frente a condição de hospitalização do bebê. As pesquisas realizadas por Barroso, Pontes e Rolim (2015) e por Chvatal, Vasconcellos, Rivoredo e Turato (2017) relatam as percepções e sentimentos maternos frente a condição de prematuridade e baixo peso de seu bebê no período de hospitalização. Ambas apresentaram abordagem qualitativa e utilizaram entrevistas e observação da linguagem corporal materna com seu bebê, como método para a coleta de dados. As entrevistas foram realizadas em salas reservadas, a mãe adolescente foi convidada a participar durante a visita ao seu bebê.

Os principais resultados da pesquisa de Barroso, Pontes e Rolim (2015) revelaram sentimentos maternos ambíguos, de tristeza e medo, e de esperança e fé em relação ao bebê; assim como do desejo de se apegar ou de indiferença frente ao bebê. Tais sentimentos mostram-se fortemente relacionados ao modo como a mãe é informada sobre o quadro de saúde e risco do bebê durante sua permanência em uma unidade de terapia intensiva neonatal. A situação da hospitalização e fragilidade do bebê pode gerar estresse familiar e consequentemente, levar a uma crise psicológica, sentimento de culpa, insegurança nos cuidados destinados ao bebê e ansiedade no enfrentamento da situação, na tentativa de adaptar-se ao novo contexto de vida.

No estudo de Chvatal, Vasconcellos, Rivoredo e Turato (2017) com mães adolescentes, os resultados apresentam sentimentos como onipotência, aflição, ambivalência, estranhamento, choque, confusão, surpresa, impotência, medo, tristeza, angústia, dor, culpa, afeto e alegria. Algumas reações de defesa também são apresentadas, como negação, dissociação, divisão (splitting) e idealização materna. A dor frente a impossibilidade de estar com o seu bebê de forma integral, por conta da hospitalização do mesmo, e de desempenhar seu papel materno refletem como os sentimentos e mecanismos de defesa se apresentam em cada participante.

Nestas duas pesquisas, os resultados permitem compreender os conflitos e a instabilidade emocional que perpassa sobre a maternidade adolescente na condição de um bebê de risco, a qual requer traçar novas perspectivas, no sentido de dar um significado ao seu papel materno e a sua identidade de mãe e mulher.

\section{Temática B - Questões psicossociais da gravidez na adolescência}

Dos 11 (onze) estudos que apresentaram desfecho perinatal o nascimento de um bebê prematuro e de baixo peso de mães adolescentes, 10 (dez) (Barroso, Pontes, \& Rolim, 2015; Chvatal, Vasconcellos, Rivoredo, \& Turato, 2017; Sámano, Martínez-Rojano, Chico-Barba, Sánchez-Jiménez, Illescas-Zarate, \& Rodríguez-Ventura, 2019; 


\section{E BEBÊ PREMATURO E DE BAIXO PESO: UMA REVISÃO INTEGRATIVA}

Santos, Costa, Amaral, Vieira, Bacelar, \& Almeida, 2014; Belfort, Santos, Pessoa, Dias, Heidelmann, \& Saunders, 2018; Hoffman, Bann, Higgins, \& Vohr, 2015; Houde, Dahdouh, Mongrain, Dubuc, Francoeur, \& Balayla , 2015; Restrepo-Méndez, Lawlor, Horta, Matijasevich, Santos, Menezes, Barros, \& Victora , 2015; Baker \& Haeri, 2014; Kirbas, Gulerman, \& Daglar, 2016) pontuaram sobre a influência distal de questões psicossociais da maternidade adolescente e que interferem diretamente nos resultados da saúde materno-infantil.

Os dados psicossociais envolvendo a maternidade adolescente seguiram a classificação da OMS - idade materna entre 10 a 19 anos, organizando as pesquisas conforme seus objetivos como mães abaixo de 16 anos (adolescência inicial) e entre 17 e 19 anos (adolescência tardia); a maioria das pesquisas apontou nível socioeconômico baixo, baixa escolaridade (ensino fundamental ou fundamental incompleto), solteira, grande parte de caracterização étnico racial negro ou hispânica, com início tardio do acompanhamento pré-natal, com maior prevalência de parto vaginal. Os bebês do estudo apresentaram baixo peso ao nascimento, menos de $2500 \mathrm{~g}$, e foram pré-termo, ou seja, nasceram antes de completar 37 semanas de gestação.

As dificuldades de acesso à informação, às condições desfavoráveis de vida e o início precoce da vida sexual sem proteção refletem sobre os prováveis impactos de uma gravidez, na maioria das vezes, não planejada.

Os principais impactos psicossociais distais, relatados nos resultados dos dez estudos foram comuns entre si. Apontam a dificuldade de retomada dos estudos, déficits nutricionais e alimentares da mãe e do bebê, possível dificuldade da participação do pai do bebê no processo, reincidência de novas gestações, rede social de apoio instável, desestruturação familiar, e situação precária de renda como reflexos sociais da maternidade adolescente. Também podem dificultar inserção da mãe adolescente nos grupos de interação, até mesmo por demandar dela um maior tempo para os cuidados com o bebê.

A precariedade de acesso a bens e serviços e de informação sobre prevenção da gravidez precoce podem interferir na maior prevalência da maternidade adolescente e dos possíveis riscos à saúde materno-infantil. Os indicadores psicossociais são essenciais para o planeamento de estratégias de prevenção em saúde e educação, pois permitem a contextualização da realidade social, que, na maior parte das culturas, atinge populações de baixa renda e de grupos sociais mais vulneráveis.

\section{Temática C - Associação entre IMC materno às taxas relativas do nascimento prematuro e/ou baixo peso do bebê}

Esta temática foi relatada nos trabalhos de Sámano et al (2019); Belfort et al (2018); Houde et al (2015) e Baker e Haeri (2014), que pontuam sobre a qualidade da alimentação materna, o peso pré-gestacional e o acompanhamento nutricional durante a gestação enquanto importantes fatores para minimizar o risco de um parto prematuro e de baixo peso ao nascimento. Estas pesquisas relatam dados estatísticos significativos da correlação entre do peso materno pré- e peri gestacional e o risco de resultados perinatais para o baixo peso e para a prematuridade do bebê.

No estudo de Sámano et al (2019), a proposta foi observar quanto o suporte familiar pode contribuir para a saúde da mãe e do bebê, analisando as características deste suporte aos resultados materno e neonatal. Entre as medidas pesquisadas, o estudo relacionou a frequência alimentar da mãe ao baixo peso do bebê. Apenas um dos quatro grupos estudados, mostrou a relação do baixo ganho de peso gestacional ao nascimento de um bebê de baixo peso, entretanto, a possível explicação deste resultado correlacionou-se a inadequação da dinâmica familiar; pois, a participação da sogra foi mais frequente do que os pais da mãe adolescente. Neste estudo observou-se que, na cultura mexicana, a presença de um forte suporte familiar é crucial para a proteção da saúde materno-infantil.

No estudo de Belfort et al (2018, uma das variáveis analisadas foi o acompanhamento nutricional durante $\mathrm{o}$ pré-natal. Os resultados indicaram que a assistência nutricional constitui uma ferramenta essencial para corrigir inadequações alimentares e controlar o ganho de peso, para uma gestação saudável e prevenção do nascimento de bebês com baixo peso.

O estudo realizado por Houde et al (2015) e por Baker e Haeri (2014), ambos desenvolvidos nos Estados Unidos, apresentaram resultados indicativos sobre um maior risco para o nascimento prematuro e de baixo peso em bebês de mães adolescentes com IMC abaixo de 30 . Os achados indicam relação entre os fatores protetores e de risco para um possível parto de um bebê prematuro e de baixo peso em adolescentes que apresentem IMC inferior ao esperado, por conta do déficit nutricional.

\section{Temática D - Risco da maternidade adolescente para a maior prevalência do nascimento de um bebê pré-termo e/ou de baixo peso e suas comorbidades}

Todos os estudos apresentados nesta pesquisa ressaltam que a maternidade durante a adolescência pode 
levar a um maior risco para o nascimento de um bebê prematuro e de baixo peso. A condição de prematuridade e baixo peso aumentam prevalência de óbitos até o primeiro ano de vida, assim como a frequentes reinternações, saúde frágil e a atrasos ou comprometimento do desenvolvimento e do comportamento da criança.

O estudo de Hoffman, Bann, Higgins \& Vohr (2015) comparou o perfil de desenvolvimento e de comportamento de bebês extremamente prematuros de mães adolescentes e de mães não adolescentes. Utilizou uma avaliação neurológica e uma escala de problemas e competência para avaliar os resultados cognitivos, de linguagem e de comportamento social em ambos os grupos. O resultado do estudo demonstrou que os aspectos relativos as alterações no desenvolvimento global são equiparadas em ambos os grupos, e os escores cognitivo, de linguagem e motor não variaram entre os grupos. Porém, os bebês de mães adolescentes eram mais propensos a apresentar problemas de comportamento, com um maior risco para resultados adversos do desenvolvimento, no campo emocional e social.

Outro estudo, desenvolvido por Botura, Bersani-Amado, Teixeira, Vituri, Caparroz-Assef e Cuman (2018), avaliou os riscos de óbito neonatal, associou a idade materna aos indicadores de prematuridade, baixo peso e morte neonatal. Os dados apontaram que quando menor a idade materna, maiores as chances de parto prematuro, bebê de baixo peso e, consequentemente, maior a ocorrência de óbito neonatal. Os resultados deste estudo são fundamentais para orientar e melhorar o atendimento para esse grupo de grávidas, com objetivo de evitar a incidência de prematuridade e baixo peso.

Vale notar que, de acordo com a análise de todas temáticas, o acompanhamento qualificado do pré-natal pode colaborar para minimizar os efeitos de complicações na gravidez adolescente e prevenir o nascimento de um bebê de forma precoce ou com peso inferior a $2500 \mathrm{~g}$.

A maternidade adolescente, por si só, não configura um risco real a saúde materno-infantil em termos biológicos. Os fatores distais e proximais de vida da mãe adolescente, como os aspectos psicossociais, relacionais, educacionais e econômicos que podem afetar a saúde do binômio mãe-bebê.

As condições de vida desfavoráveis foram pontuadas enquanto agravante em todos os estudos, independente dos padrões culturais e econômicos dos países em que estes foram desenvolvidos.

Quando a maternidade adolescente está associada ao risco psicossocial vigente, enfatiza-se a necessidade de considerar a gravidez na adolescência como um problema de saúde pública. O risco de desdobramentos neonatais prejudiciais à saúde materno-infantil deve ser considerado.
Neste sentido, intensificar ações e estratégias eficazes de políticas de saúde e educação, que considerem o cenário sociocultural e a individualidade do "adolescer" e do "maternar", devem minimizar possíveis complicações da gravidez precoce e controlar as taxas de nascimento prematuro e de baixo peso (Botura et al, 2018). Estratégias como planejamento familiar, saúde sexual e reprodutiva, apresentam se como alternativas importantes de prevenção e promoção da saúde materno-infantil (Santos, Lara, Lima, Rocha, Rocha, Glória \& Ribeiro, 2018).

\section{Considerações Finais}

Esta pesquisa teve como objetivo realizar um levantamento dos estudos que fazem correlação entre a maternidade adolescente e os resultados do nascimento prematuro e com baixo peso.

Entre as análises dos estudos, foram observados trabalhos de abordagem qualitativa, que buscaram refletir as questões psicoemocionais da mãe adolescente frente a um bebê de risco, assim como trabalhos de caráter quantitativo e retrospectivo, que buscaram demonstrar estatisticamente as correlações entre a ocorrência da gravidez adolescente ao parto prematuro e/ou de um bebê com baixo peso ao nascimento.

Os resultados demonstraram diferentes variáveis que acarretam riscos para a mãe adolescente e seu bebê, especialmente porque as duas condições ampliam os efeitos negativos para a saúde de ambos e que estão diretamente ligados ao aumento do risco psicossocial e de desenvolvimento da criança.

Embora os estudos abordem temáticas diversas, a maternidade adolescente, por si só, não predispõe ao nascimento de um bebê pré-termo e de baixo peso. A análise dos resultados das pesquisas aponta uma correlação multifatorial, que depende de aspectos relacionados ao contexto de vida e saúde da adolescente; assim como de fatores sociais e culturais.

Entender o cotidiano das relações estabelecidas pela adolescente e investir em ações de saúde e educação são estratégias preliminares para a prevenção de uma gravidez precoce, que muitas vezes são indesejadas.

O apoio familiar e social, bem como hábitos de vida saudável na instancia de uma maternidade adolescente, podem colaborar com resultados mais positivos para o cuidado em saúde da mãe e do bebê.

Por se tratar de uma situação peculiar, este ensaio teórico sugere a necessidade de maior atenção a estudos que abordem a correlação da maternidade adolescente e o bebê de baixo peso e pré-termo, especialmente sob a ótica multidisciplinar, cultural e familiar, tangenciando os 


\section{CORRELAÇÕES ENTRE MATERNIDADE NA ADOLESCÊNCIA E BEBÊ PREMATURO E DE BAIXO PESO: UMA REVISÃO INTEGRATIVA}

possíveis desdobramentos dos impactos sobre a saúde materno infantil no decorrer de sua vida após a alta hospitalar e subsidiando estratégias em saúde para prevenção de agravos psicossociais e de saúde da mãe e do bebê.

\section{Referências}

Asnong, C., Fellmeth, G., Plugge, E., Wai, N.S., Pimanpanarak, M., Paw, M.K., Charunwatthana, P., Nosten, F., \& McGready, R. (2018). Adolescents' perceptions and experiences of pregnancy in refugee and migrant communities on the Thailand-Myanmar border: a qualitative study. Reprod Health 15, 83. https://doi.org/10.1186/s12978-018-0522-7

Azevedo, W.F., Diniz, M.B., Fonseca, E.S.V.B., Azevedo, L.M.R., \& Evangelista, C.B. (2015). Complicações da gravidez na adolescência: revisão sistemática da literatura. Einstein, 13(4):618-26.

Baker, A.M., \& Haeri, S. (2014). Estimating risk factors for spontaneous preterm delivery in teen pregnancies. Arch Gynecol Obstet, 289:1203-1206 DOI 10.1007/s00404-014-3149-0.

Barroso, M.L., Pontes, A.L., \& Rolim, K.M.C. (2015). Consequências da prematuridade no estabelecimento do vínculo afetivo entre mãe adolescente e recém-nascido. Revista da Rede de Enfermagem do Nordeste, vol. 16, núm. 2, março-abril, pp. 168 - 175.

Becker, Daniel (1993). O que é adolescência? (10ª Ed.) [eBook, 2017] São Paulo: Editora Brasiliense. 104 p.

Belfort, G.P., Santos, M.M.A.S., Pessoa, L.S., Dias, J.R., Heidelmann, S.P., \& Saunders, C. (2018). Determinantes do baixo peso ao nascer em filhos de adolescentes: uma análise hierarquizada. Ciência \& Saúde Coletiva, 23(8): 2609-2620.

Botura, C.A., Bersani-Amado, L.E., Teixeira, J.J.V., Vituri, S.C., Caparroz-Assef, S.M., \& Cuman, R.K.N. (2018). Risk factors for mortality in Neonatal Intensive Care Unit: maternal age influence. Acta Scientiarum. Health Sciences, v. 40, e32717.

Chvatal, V. L. S., Vasconcellos, J. F. J., Rivoredo, C. R. S., \& Turato, E. R. (2017). Mecanismos de Defesa Utilizados por Adolescentes com Bebês Prematuros em UTI Neonatal. Paidéia, Vol. 27, Suppl. 1, 430-438. doi:10.1590/1982-432727s1201708.

Flores-Valencia, M.E., Nava-Chapa, G., \& Arenas-Monreal, L. (2017). Embarazo en la adolescencia en una región de México: un problema de Salud Pública. Rev. Salud Pública, 19 (3): 374-378.

Hoffman, L., Bann, C., Higgins, R., \& Vohr, B. (2015). Developmental Outcomes of Extremely Preterm Infants Born to Adolescent Mothers. Pediatrics Volume 135, number 6, June, 1082-92.

Houde, M., Dahdouh, E.M., Mongrain, V., Dubuc, E., Francoeur, D., \& Balayla, J. (2015). The effect of adequate gestational weight gain among adolescents relative adults of equivalent body mass index and the risk of preterm birth, cesarean delivery and low birth weight. I Pediatr Adolesc Gynecol 28, 502-507.

Kane, S., Miedema, E., Dielemana, M., \& Broerse, J. (2019). You have a child who will call you "mama"': understanding adolescent pregnancy in South Sudan. Global Health Action, 12:1, DOI: 10.1080/16549716.2018.1553282

Kawakita, T., Wilson, K., Grantz, K.L., Landy, H.J., Huang, C., \& GomesLobo, V. (2016). Adverse maternal and neonatal outcomes in adolescent pregnancy. Pediatr Adolesc Gynecol 29 (2016) 130e136.

Kirbas, A., Gulerman, H.C., \& Daglar, K. (2016). Pregnancy in Adolescence: Is it na obstetrical risk? J Pediatr Adolesc Gynecol, 29, 367e371.

Lakshmanan, A., Agni, M., Lieu, T., Flugler, E., Friedlich, O.S., McCormick, M.C., \& Belfort, M.B. (2017). The Impact of Preterm Birth $<37$ Weeks on Parents and Families: a cross sectional study in two years after discharge from the neonatal intensive care unit. Health and Quality of Life Outcomes, 15:30.

Leal, M.C., Esteves-Pereira, A.P., Nakamura-Pereira, M., Torres, J.A., ThemeFilha, M., Domingues, R.M.S.M., Dias, M.A.B., Moreira, M.E., \& Gama, S.G. (2016). Prevalence and risk factors related to preterm birth in Brasil. Reproductive Health, 13(Suppl 3):127 DOI 10.1186/s12978-016-0230-0
Machado Jr, L.C., Passini Jr, R., \& Rosa, I.R.M. (2014). Late prematurity: systematic review. J Pediatr, Rio de Janeiro, 90(3):221-31. doi:10.1016/j. jped.2013.08.012.

Moher D, Liberati A, Tetzlaff J, Altman DG, The PRISMA Group (2009). Preferred Reporting Items for Systematic Reviews and Meta-Analyses: The PRISMA Statement. PLoSMed 6(7).

Mota, L.A., Sá, F.E. de, \& Frota, M.A. (2005). Estudo Comparativo de Desenvolvimento Sensório-Motor de Recém-Nascidos Prematuros da Unidade de Terapia Intensiva Neonatal e do Método Canguru. RBPS, 18(4): 191-198.

Ncube, R.K., Barlow, H., Mayers, P.M. (2016). A Life Uncertain - My Baby's Vulnerability: mothers lived experience of connection with their pretem infants in a Botswana neonatal intensive care unit. Curationis, 39(1), a1575.

Ochen, A.M., Chi, P.C., \& Lawoko, S. (2019). Predictors of teenage pregnancy namong gilrs age 13-19 years in Uganda: a community based case-control study. BMC Pregnancy and Childbirth, 19:211.

Oliveira R. R., Santos, S.S.C. \& Melo, E.C. (2016). Nascimento prematuro e assistência pré-natal: revisão integrativa à luz de Canguilhem. Rev Fund Care Onlin; 8(3), 4616-4622.

Restrepo-Méndez, M.C., Lawlor, D.A., Horta, B.L., Matijasevich, A., Santos, I.S., Menezes, A.M.B., Barros, F.C., \& Victora, C.G. (2015). The Association of Maternal Age with Birthweight and Gestational Age: A Cross-Cohort Comparison. Paediatric and Perinatal Epidemiology, 29, 31-40

Sámano, R., Martínez-Rojano, H., Chico-Barba, G., Sánchez-Jiménez, B., Illescas-Zarate, D., \& Rodríguez-Ventura, A.L. (2019). Characteristics of the Family Support Network of Pregnant Adolescents and Its Association with Gestational Weight Gain and Birth Weight of Newborns. Int. J. Environ. Res. Public Health, 16, 1222; doi:10.3390/ijerph16071222.

Santos, L. A. V., Lara, M. O., Lima, R. C. R., Rocha, A. F., Rocha, E. M., Glória, J. C. R., \& Ribeiro, G. D. C. (2018). História gestacional e características da assistência pré-natal de puérperas adolescentes e adultas em uma maternidade do interior de Minas Gerais, Brasil. Ciência \& Saúde Coletiva, 23, 617-625

Santos, N.L.A.C., Costa, M.C.O., Amaral, M.T.R., Vieira, G.O., Bacelar, E.B., \& Almeida, A.H.V. (2014). Gravidez na adolescência: análise de fatores de risco para baixo peso, prematuridade e cesariana Ciência \& Saúde Coletiva 19(3):719-726, 2014

Santos, R.C.A.N., Silva, R.M., Queiroz, M.V.O., Jorge, H.M.F., Brilhante, A.V.M. (2018). Realidades e perspectivas de mães adolescentes acerca da primeira gravidez. Rev Bras Enferm [Internet], 71(1):65-72.

Shahabuddin, A., Nostlinger, C., Delvaux, T., Sarker, M., Delamou, A., Bardaj1, A., Broerse, J.E.W., \& Brouwere, V.D. (2017) Exploring Maternal Health Care-Seeking Behavior of Married Adolescent Girls in Bangladesh: A Social-Ecological Approach. PLoS ONE 12(1): e0169109.

Silveira, M.F., Matijazevit, A., Horta, B., \& Bettiol, H. (2013). Prevalência de Nascimentos Pré-termo por Peso ao Nascer: revisão sistemática. Rev Saúde Pública, 47 (5), 992-1000.

Sychareun, V., Vongxay, V., Houaboun, S., Thammavongsa, V., Phummavong-

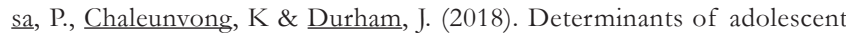
pregnancy and access to reproductive and sexual health services for married and unmarried adolescents in rural Lao PDR: a qualitative study. $B M C$ Pregnancy Childbirth, 18:219.

Tiba, Içami (2010). Adolescentes: quem ama, educa! São Paulo: Integrare Editora. Ursi, E. S. (2005). Prevenção de lesões de pele no perioperatório: revisão integrativa da literatura. Dissertação de mestrado. Ribeirão Preto: Universidade de São Paulo, Escola de Enfermagem de Ribeirão Preto. Apud Souza, M.T., Silva, M.D., \& Carvalho, R. (2010). Revisão integrativa: o que é e como fazer. Einstein. 8 (1 Pt 1):102-6

Von Grafen, B.V., Gomes, J.S., Lorenzoni, A.M.C., Strassburger, S.Z., Ferreira, P.F., \& Campanaro, M.P. (2017). Fatores de Risco para Nascimentos Pré-Termo em uma Unidade de Terapia Intensiva Neonatal. Unijuí, in XXII Jornada de Pesquisa.

Submetido em: 10-6-2020

Aceito em: 17-8-2020 